\title{
An immunoassay cassette with a handheld reader for HIV urine testing in point-of-care diagnostics
}

\author{
Wenbo Yang ${ }^{1}$ - Dianlong Yang ${ }^{1} \cdot$ Shisong Gong ${ }^{1} \cdot$ Xiaobing Dong ${ }^{1} \cdot$ Luyao Liu ${ }^{1} \cdot$ Shengda Y ${ }^{1}{ }^{1} \cdot$ Xiaolei Zhang $^{2}$. \\ Shengxiang $\mathrm{Ge}^{3} \cdot$ Dong Wang ${ }^{2} \cdot$ Ningshao Xia $^{3} \cdot$ Duli $\mathrm{Yu}^{1,4} \cdot \mathrm{Xianbo}^{\mathrm{Q}} \mathrm{Qu}^{1}$
}

Published online: 21 May 2020

(C) Springer Science+Business Media, LLC, part of Springer Nature 2020

\begin{abstract}
Currently, most HIV tests are performed with blood samples, or alternatively saliva samples are used for HIV testing. Simple HIV tests need to be performed in hospitals or other medical agencies instead of more invasive HIV blood tests. To enable point-ofcare (POC) HIV diagnostics, based on a recently developed lateral flow strip for HIV urine testing, a microfluidic immunoassay cassette with a handheld optical reader is developed. Based on lateral flow strip with gold colloid reporter, the integrated immunoassay cassette can perform sample introduction, metering, discharging, applying and detection which simplifies HIV testing. An indicator is incorporated into the cassette to guide sample introduction based on color change, and further, the excess test sample is stored inside the sealed cassette to avoid any contamination. The low-cost handheld optical reader can provide a test result within a few seconds, which is useful for simple, sensitive and affordable HIV onsite detection. Instead of using normal white LEDs, a customized back light module embedded with green LEDs is adopted to illuminate the lateral flow strip with an appropriate working current to achieve optimal performance. Compared to the standard lateral flow strips using a benchtop reader, with the disposable immunoassay cassette assisted by the handheld optical reader, more convenient, easier-to-operate, and more affordable HIV urine testing can be achieved in POC diagnostics.
\end{abstract}

Keywords HIV test · Urine $\cdot$ Immunoassay $\cdot$ Lateral flow strip $\cdot$ Microfluidic cassette $\cdot$ Handheld optical reader $\cdot$ Point-of-Care (POC) diagnosis

\section{Introduction}

Acquired Immune Deficiency Syndrome (AIDS) is still a serious global disease since it cannot be cured completely. For people infected with HIV, AIDS is a stage when the level of their CD4 + T lymphocytes falls below acritical, for example 200 cells $/ \mu \mathrm{L}$ (Cheng et al. 2009). By 2018, it was estimated that the global number of people living with HIV was $\sim 37.9$ million and

Xianbo Qiu

xbqiu@mail.buct.edu.cn

1 Institute of Microfluidic Chip Development in Biomedical Engineering, College of Information Science and Technology, Beijing University of Chemical Technology, Beijing 100029, China

2 Beijing Wantai Biological Pharmacy Enterprise Co. Ltd., Beijing 102206, China

3 National Institute of Diagnostics and Vaccine Development in Infectious Diseases, Xiamen University, Xiamen 361005, China

4 Beijing Advanced Innovation Center for Soft Matter Science and Engineering, Beijing 100029, China more than two-thirds of them reside in developing countries (World Health Organization 2019; Vos et al. 2016). There are common methods for HIV detection based on different principles (Qiu et al. 2009, 2011; Chen et al. 2016). Using an immunoassay, HIV can be diagnosed by detecting anti-HIV antibody or HIV viral antigen. Based on molecular analysis, HIV can be diagnosed by detecting viral nucleic acids. Because HIV molecular diagnostic viral testing needs complicated sample processing for nucleic acid extraction and elaborate nucleic acid amplification, it is difficult to perform molecular diagnosis in developing countries where medical infrastructural facilities are lacking (Zhao et al. 2019).

Among different detection methods based on HIV immunoassay, lateral flow strips are widely used for on-site screening tests, especially in resource-poor settings at POC because of their ease of use, low-cost, simplicity and rapidity (Granade et al. 2010). Various types of lateral flow strips have been developed for HIV immunoassays (Paredes et al. 2006). For example, anti-HIV antibody is can be detected in serum samples using lateral flow strips (Faulstich et al. 2007). In principle, the detection result can be observed by eyes when the gold 
colloid reporter lateral flow strips are used. To avoid the interference from red blood cells, the raw whole blood sample needs to be centrifuged to obtain the cell-free serum sample. Alternatively, an additional filtration pad adjacent to the sample pad can be incorporated into the lateral flow strip to block the red blood cell in lateral flow assay. HIV saliva tests can also be performed with lateral flow strip (Delaney et al. 2006). Compared to HIV blood testing, HIV detection can be implemented much more conveniently with saliva samples. To improve detection sensitivity, up-converting phosphor (UCP) reporter particles have been used to label the HIV antibody and avoid interfering background (Liu et al. 2009). In this case, a companion optical reader is required to perform HIV saliva test with lateral flow strip when fluorescence labeling is adopted, which may partly limit its application field.

Urine testing is a routine examination in vitro-diagnosis. In fact, because of the superiority with noninvasive collection of samples, urinalysis is becoming a competitive format in POC diagnosis as more diagnostic biomarkers are identified in urine (Mahoney et al. 2020; Hart et al. 2011). For example, as an emerging application of urine analysis, in situ monitoring of Activities of Daily Living (ADL) is able to provide systematical information of the body status with continuous multi-parameter measurements of urine in a home setting for POC testing (Taramasco et al. 2018). Traditionally, urinalysis is able to provide not only valuable clinical information for diagnosis of various urologic and renal diseases, but also evidence in asymptomatic patients, for example, with sexually transmitted infections (STI's) (Fogazzi and Perazella 2010). In POC diagnosis, widely used urinalysis devices include dip strips or sticks for pregnancy testing, urinary tract infections (UTIs), and urine test strips for other types of analytes of urine samples, for example, Specific Gravity, Creatinine, Glucose, Ions, Ketones, Lactate, Nitrite, $\mathrm{pH}$, Protein, and Uric Acid (Kingston et al. 2003; Suresh et al. 2018). For high throughput detection, DNA microarrays can be used for simultaneous detection of different DNA markers in urine (Gaber et al. 2017). To avoid the inconvenience with urine sample storage and transportation, POC diagnosis devices, which can be easily implemented at the clinic or even at home, are highly desired in urine testing. With simple, convenient and easyto-handle operation, POC urinalysis devices developed based on microfluidic or lab-on-chip technology are able to detect infectious diseases or monitor chronic diseases with only modest resources (Lepowsky et al. 2017; Jalal et al. 2017). Compared to blood testing requiring comparatively large sample volumes, POC microfluidics-based urine testing is quite promising and attractive for home use on account of the noninvasive nature of urine sample collection (Lin et al. 2011).

To effectively control HIV epidemics, sensitive, simple, low-cost and easy-to-use detection methods need to be developed for rapid HIV test in POC settings. In this article, we describe a microfluidic immunoassay cassette with a companion handheld optical reader for HIV test based on urine sample analysis. Based on a recently developed gold colloid lateral flow strip available for HIV urine testing, a disposable immunoassay cassette is developed to perform self-assisted urine metering, discharging, applying and detection. A low-cost handheld optical reader is developed to detect the test result from the cassette. Once the raw, undiluted urine sample is loaded into the cassette, lateral flow immunoassay can be easily performed by the untrained operators themselves. Excess sample will be retained inside the cassette to prevent any environmental contamination. The affordable handheld optical reader with a total component cost of seventy dollars improves the detection sensitivity, and logs, displays and transmits the test result if necessary. The performance of both the cassette and the handheld optical reader is systematically evaluated. Experimental results show that successful HIV urine testing can be conveniently performed in POC settings with the test reported here.

\section{Material and methods}

\subsection{Lateral flow immunoassay for HIV test with urine sample}

Based on the immune specificity of HIV viral surface antibodies, an easy-to-operate, one-step lateral flow immunoassay was developed for HIV-1 antibody test with urine sample. For each test, the applied volume of urine sample is $\sim 80 \mu \mathrm{L}$. Similar to existing lateral flow strips (Qian and Bau 2004), as shown in Fig. 1a, the HIV urine test strip $(60 \mathrm{~mm} \times 4 \mathrm{~mm})$ is comprised of a backing, sample pad, conjugate pad, nitrocellulose membrane, and absorbent pad. On the conjugate pad, staphylococcal protein A (SPA) conjugated to gold colloid nanoparticles is stored in dry form after diluted in a blocking reagent with bovine serum albumin (BSA). Witnessing the fact of low concentration of biomarkers with urine sample, instead of using the sandwiched immunoassay which normally adopted in HIV blood testing, indirect immunoassay is adopted in HIV urine testing to ensure the detection sensitivity by capturing HIV antibody from urine sample through SPA. Moreover, to improve the detection sensitivity, large sample volume, e.g., $80 \mu \mathrm{L}$ is adopted in $\mathrm{HIV}$ urine testing. In the test line area of the nitrocellulose membrane, HIV antigens are immobilized to form the capture line. In the control line area, the second antibodies are immobilized to form the verification line.

Once the urine sample is in contact with the sample pad of the lateral flow strip, it is wicked along the lateral flow strip by capillary force. At most 15 min later, the result becomes readable by observing the test or control line with red color. As shown in Fig. 1b, in a predefined sequence based on indirect immunoassay, first the HIV antibodies in the test urine sample 
Fig. 1 Lateral flow immunoassay strip for HIV urine testing
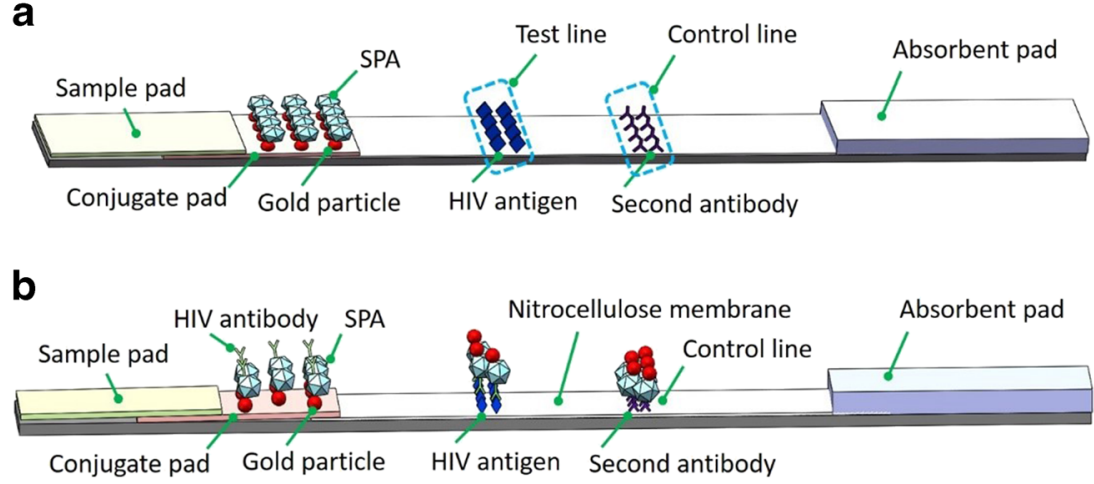

bind to the gold colloid particles stored on the conjugate pad through SPA, and then they move together along the strip until captured by the test line based on the specific immune bioreactivity between the immobilized HIV antigens and the detected antibodies. Finally, the remaining unbound free gold colloid particles are captured by the control line area for assay verification through the binding reaction between SPA and the second antibody. The lateral flow strip recently developed by Beijing Wantai Biological Pharmacy Enterprise Co (Beijing, China) for HIV urine testing has been evaluated thoroughly and systematically using a large sample bank for clinical trials. It has demonstrated $98.4 \%$ of accuracy with the HIV positive subjects without antiretroviral therapy (ART), and 100\% specificity with HIV negative subjects (Beijing Wantai 2019). Compared to HIV blood testing, as a noninvasive detection method, HIV urine testing can be performed more easily and conveniently, which is suitable for self-test in POC settings. It is well known that HIV saliva test is a commercialized noninvasive detection method. Compared to HIV salvia testing, HIV urine testing can be performed with relatively simpler procedure for sample collection and pre-treatment. However, the unfriendly smell from the urine sample could be one of the disadvantages with HIV urine testing. Nevertheless, similar to HIV saliva test, HIV urine testing provides another choice for noninvasive HIV POC test.

\subsection{Immunoassay cassette with self-assisted urine metering, discharging and applying}

In POC diagnosis, to allow on-site HIV urine tests with the lateral flow strip performed by laymen instead of trained operators for example for home use testing, an immunoassay cassette with the capability of sample metering, discharging and applying was developed. Figure 2 depicts the immunoassay cassette equipped with a lateral flow strip. As shown in Fig. 2a, the immunoassay cassette consists of three major modules, e.g., sample introduction module on the top, sample metering module in the middle, and detection module on the bottom.
The sample introduction module consists of a $30 \mathrm{~mm} \times$ $20 \mathrm{~mm} \times 6 \mathrm{~mm}$ poly(methyl methacrylate) (PMMA) loading chamber whose top is partly covered with a PMMA layer. As shown in Fig. 2a, the urine sample from a normal container can be directly added into the sample introduction module through the loading port. An accessory tape layer on top is used to seal the loading port after sample loading, which reduce the risk of environmental contamination. The sample metering module consists of a $30 \mathrm{~mm} \times 40 \mathrm{~mm} \times 8 \mathrm{~mm}$ PMMA storage chamber with a vent hole on top. As shown in Fig. $2 \mathrm{a}$, in the center of the storage chamber, there has an independent metering chamber with a size of $\sim 80 \mu \mathrm{L}$. Meanwhile, the space around the metering chamber in the storage chamber is used as the overflow chamber to hold the excess urine sample. An absorbent paper layer (Nanjing Huiyuechi Electronic Technology Company Ltd.) is deposited into the overflow chamber to not only absorb any excess urine sample, but also to work as an indicator for sample loading since its color will obviously change from white to red on contact with urine. Opposite to the metering chamber, an extruded connection head is fabricated on the bottom of the sample loading chamber to allow urine sample to smoothly enter into the metering chamber. The metering chamber also has an extruded head to establish a flow path to the lateral flow strip once the device is fully assembled. Initially, the bottom of the extruded head is sealed with a thin $4 \mathrm{~mm} \times 4 \mathrm{~mm}$ paper layer (Shenzhen Siweituoda Technology Company Ltd.). The specific thin paper layer, which is made from stretched polytetrafluoroethylene (PTFE) with average pore sizes from 0.1 to $1.5 \mu \mathrm{m}$, has an average pore density larger than $80 \%$. The specific thin paper layer is able to hold the urine sample inside the metering chamber while allowing air to pass through. The detection module consists of a lateral flow strip, a strip holder, and a sharp needle partly penetrating into the strip from the sample pad. To simplify the process of fabrication, different parts of the cassette were all cut with a laser machine (HTC0906-W80, Jinan Hantong Digital Control Device Enterprise Co. Ltd., Jinan), and then laminated with the double-sided tape (Type \#9731, 3 M). To reduce the complexity of cassette fabrication and binding, before laser cutting, the PMMA sheet was first laminated with the double-sided tape from one side to allow 
Fig. 2 HIV immunoassay cassette: (a) an exploded view of the integrated cassette with a gold colloid lateral flow strip; (b) a photograph of the fabricated cassette a

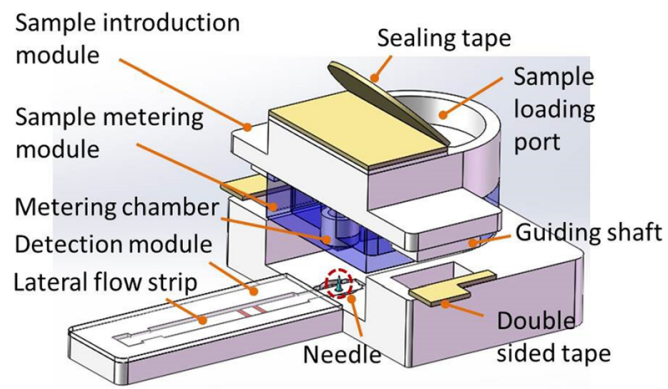

b

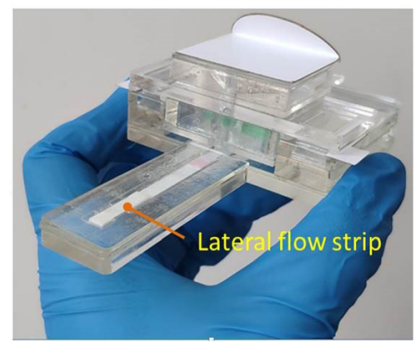

both of them to be cut simultaneously, which was helpful to improve the binding performance.

Initially, the sample introduction module has been bond with the sample metering module with double-sided tape, and the combined two modules are partly assembled with the detection module through two guiding shafts from both sides (as shown in Fig. 2a). After sample loading, the two combined modules can be conveniently assembled with the bottom detection module by hand. For safety, two doublesided tape layers on both sides are used to combine and fix three modules together after assembling (as shown in Fig. 2a). During assembly, the bottom needle (as shown in Fig. 2a) sitting on the detection module will penetrate through the thin paper layer under the sample metering chamber, which allows the urine sample to be continually imbibed by the sample pad of the lateral flow strip for immunoassay. As shown in Fig. 2b, the top window of the detection module is covered by a transparent PMMA layer for optical detection.

\subsection{Handheld optical reader}

A handheld optical reader $(66 \mathrm{~mm} \times 66 \mathrm{~mm} \times 66 \mathrm{~mm})$ with low-cost was developed to read and analyze the test result from the lateral flow strip, which allows the test result to be saved, displayed and transmitted if necessary. In principle, with the handheld optical reader, more sensitive, accurate and consistent test result can be achieved compared to typical eye observation which can be affected by a couple of issues. As shown in Fig. 3a, a small-size CMOS camera module (openmv3 cam m7, OpenMV, LLC) equipped with an optical lens was used to collect image from the lateral flow strip when it was illuminated by a back light module from the top. Since the gold nanoparticles have a peak wavelength of absorbing light around $525 \mathrm{~nm}$, green light from the back light module consisting of green LEDs (wavelength: 510-525 nm) was adopted to illuminate the lateral flow strip from top to improve the detection sensitivity. A circuit module consisting of a single-chip microcontroller (N76E003AT20, Nuvoton Technology Corporation) was used to control the camera module to collect and analyze image, and then the test result was sent to the LCD module for display. A Bluetooth module was connected to the single-chip microcontroller for data transmission. To reduce system power-consumption, instead of using an advanced camera, a compact camera module relying on a micro-controller based on ARM7 (STM32F407, STMicroelectronics) was adopted, which was able to perform image processing with a built-in function library based on Micro Python after image collection. Especially, for the image collection and processing module, a micro-controller with significantly low power consumption was adopted, which was critical to reduce the system total power consumption. The system currents for idle and working states are $160 \mathrm{~mA}$ and $90 \mathrm{~mA}$, respectively. Powered by two $1.5 \mathrm{~V}$ batteries, the device is able to continually work up to $4 \mathrm{~h}$.

Figure $3 \mathrm{c}$, d, respectively, depict the optical reader with a partly- and fully-inserted immunoassay cassettes. For the HIV urine test, once the urine sample is applied onto the lateral flow strip, the test result becomes detectable after $15 \mathrm{~min}$. With the companion hand-hold optical reader, rapid, on-site, HIV urine testing with lateral flow immunoassay can be conveniently performed at home or in other POC settings. The total cost of components of the hand-hold optical reader is around seventy dollars, which is beneficial to POC diagnosis.

\subsection{Image analysis for lateral flow strip detection}

A custom algorithm was developed to analyze the test, control lines and the background of the lateral flow strip to achieve the detection result for HIV urine testing. As shown in Fig. 4a, the original image was captured by the CMOS camera in the handheld optical reader with the resolution of $640 \times 480$ pixels. As shown in Fig. 4b, an interest area was extracted from the original image for further analysis, and then it was converted into a gay image (Fig. 4c). As shown in Fig. 4d, to improve the detection sensitivity, Laplacian operator was used to process the gray image to strengthen the boundary between different parts of the lateral flow strip (Tian et al. 2020).

There are three major steps for image processing of the lateral flow strip. Similar to the test or control line, a background line is adopted here to represent the background area of the lateral flow strip. Because of the limited resolution of the captured image, the test, control and background lines are 
Fig. 3 Handheld optical reader: (a) schematics of the handheld optical reader; (b) a photograph of the handheld optical reader; (c-d) a handheld optical reader respectively with a partly- and fully-inserted HIV immunoassay cassette a

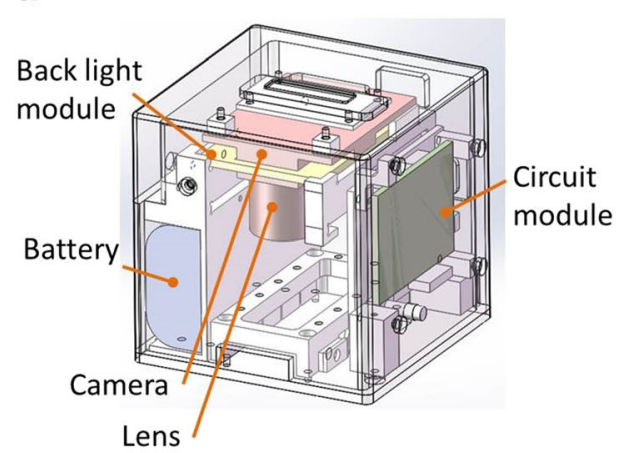

C

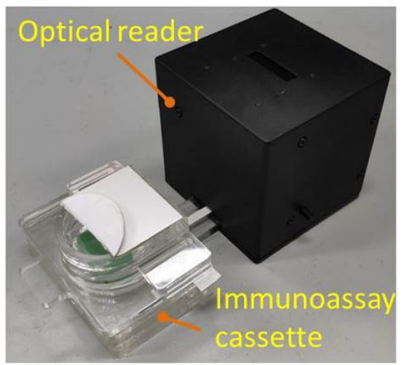

b

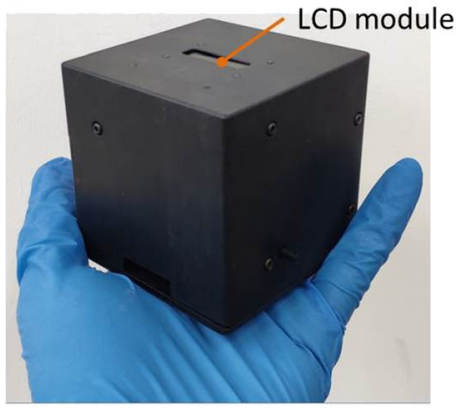

d

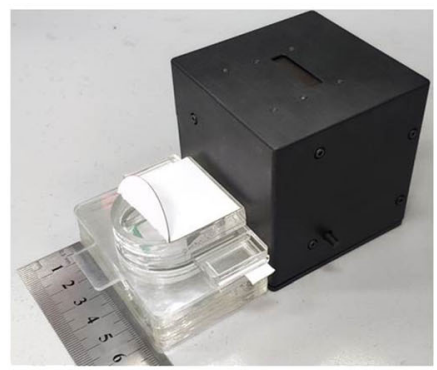

all regarded as a short line $(g(x, y))$ with a fixed height of one pixel. First is to search the control line within the image processing domain (Fig. 4e). The location of the control line is identified by analyzing the difference of the mean gray (graymean) between the control line and its adjacent area. To reduce the time required by image processing, the boundaries of the searching area are defined as $y=y_{c} \pm n(1<n \leq 5)$. Here, $y_{c}$ is the estimated location of the control line.

As shown in Fig. 4f, gray mean at different locations, for example, $y=y_{c}$ or in between $y=y_{c} \pm n(1<n \leq 5)$ can be calculated by Eq. (1):

$\operatorname{gray}_{\text {mean }}=\frac{1}{2 n} \sum_{i=1}^{2 n} g_{i}(x, y)$
The control line can be identified by comparing the difference of the mean gray between $\left(y=y_{c}\right)$ and $\left(y=y_{c} \pm n(1<n \leq\right.$ 5)) with a predefined threshold when the image processing domain is scanned with different $y=y_{c}$. Here, the threshold was set to 10 .

Within the image processing domain (Fig. 4e), in the $x$ direction, the searching range of image processing domain is within $W=\left|\left(\frac{w}{2}+n\right)-\left(\frac{w}{2}-n\right)\right|$, and in the $y$ direction, it is between $y=y_{0}+\frac{h}{2}$ and $y=y_{0}+h$. In the $y$ direction, the searching range is limited to the lower half part of the domain to reduce the time required for image processing.

The second step is to identify the test line. Based on the location of the control line, similar algorithm can be used to find the test line within a specific image processing domain.
Fig. 4 Image analysis of the latera flow strip: (a) captured original image; (b) interest area of the original image; (c) the converted gray image; (d) image processed with Laplacian operator; (e) image processing domain; (f) schematics of specific target searching and gray calculation

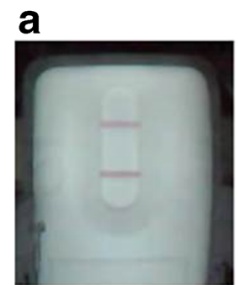

b

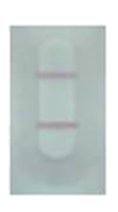

c

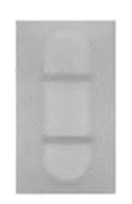

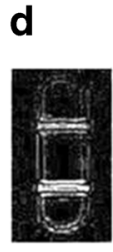

e

$\left(x_{0}, y_{0}\right)$

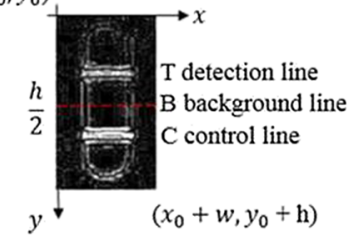

f $y=y_{c}-n$
$y=y_{c}+n$ 
Third, the middle area between the control and test lines can be identified as the background line. To compensate the test fluctuation coming from the device or the process of detection, the gray difference between the test and the background lines (relative gray) is adopted as the calibrated result. Finally, the detection result for HIV urine testing with lateral flow strip can be achieved by comparing the relative gray of the test line with a predefined threshold.

\section{Results and discussion}

Urine samples from healthy donors were collected by National Institute of Diagnostics and Vaccine Development in Infectious Diseases (NIDVD) of Xiamen University with relevant guidelines and regulations approved by the Ethics Committee of the NIDVD and stored in $1.5 \mathrm{~mL}$ collection tubes before use. All fresh urine samples were used within 30 min after collection. HIV serum sample with a high concentration was provided by Beijing Wantai. The original HIV serum sample was diluted with serum to achieve multiple test samples with different concentrations. HIV antibodies from Beijing Wantai were spiked in healthy urine to simulate HIV positive urine samples. The original HIV urine sample was diluted with urine to achieve multiple test samples with different concentrations.

\subsection{Urine metering, discharging and applying with immunoassay cassette}

As described above, in each test, the urine sample was first loaded into the metering chamber, and then discharged and applied onto the lateral flow strip for immunoassay. First, water was used as the test sample for performance evaluation of metering. In each experiment, approximately $300 \mu \mathrm{l}$ of water was loaded into the cassette, and after a couple of seconds, the stored water from the metering chamber was weighted with a precise balance (FA1004B, Shanghai Yoke Instrument $\mathrm{CO}$. Ltd.) to determine its volume based on its density $\left(1 \mathrm{~g} / \mathrm{cm}^{3}\right)$. Parallel experiments with urine samples were performed. Next, experiments for performance evaluation of discharging and applying were also performed. Similarly, both water and urine were used as the test samples. In each experiment, approximately $300 \mu \mathrm{l}$ of water or urine was loaded into the cassette. First, the detection module of the cassette was weighted with a precise balance before sample discharging. Secondly, after the sample was discharged into the detection module within a couple of min, the detection module was weighted again with a precise balance. Based on the sample density, the volume of the discharged sample can be estimated by comparing the two weights of the detection module before and after sample discharging. Figure 5a depicts the measured sample volume respectively from metering or discharging in different experiments.

As shown in Fig. 5a, it can be seen that either with water or urine, the volume deviation of the metered or discharged sample is within $80 \pm 8 \mu \mathrm{l}$, which is acceptable for HIV lateral flow immunoassay. As shown in Fig. 5b, the indicative absorbent paper works well since after sample loading, its color changes from white to red, which is helpful to guide proper operation.

\subsection{Performance evaluation of handheld optical reader}

In principle, the gold nanoparticles have a peak wavelength of absorbing light around $525 \mathrm{~nm}$. Therefore, to optimize the system performance, different light sources including white LED and green LED were used to illuminate the lateral flow strip for comparison. To uniformly illuminate the entire detection area of the lateral flow strip for consistent performance, instead of directly using two independent LEDs as point light sources, a customized $33 \mathrm{~mm} \times 27 \mathrm{~mm}$ back light module (Shenzhen Baohui Photoelectricity Company Ltd.) was adopted. In the back light module, the light from multiple embedded white or green LEDs enters into a planar light waveguide from the side wall, which is able to provide uniform illumination. In all experiments, the driving current of the back light module was appropriately set to $1.5 \mathrm{~mA}$. Standard gold colloid lateral flow strips, instead of the developed cassette, were used to evaluate the performance of the handheld reader itself. HIV serum samples with different concentrations were used in experiments. In each test, $80 \mu \mathrm{l}$ of HIV serum sample was applied onto the standard lateral flow strip specific for HIV serum detection and incubated for 15 min. Figure 6 depicts the experimental results from two back light modules with different colors. Error bars come from multiple repeated experiments. The signal amplitude is defined as the signal intensity difference between the test line and the background.

As shown in Fig. 6, it can be found that with green LEDs, optimal performance can be achieved compared to white LEDs. Inserts show photographs of the standard lateral flow strips. As shown in the insert of Fig. 6, a specific number (\#) is assigned to each lateral flow strip based on a customized rule to represent the signal intensity of the test line. From \#2 to $\# 10$, they respectively correspond to low-positive to high-positive. For the described handheld optical reader, low-positive detection close to \#3 is the acceptable limit of detection. In fact, it is difficult for a person to locate the test line from the background for \#3 low-positive detection just by eyes. Therefore, compared to traditional eye observation, the handheld optical reader is helpful to improve the detection sensitivity of HIV urine test with lateral flow strip. As shown in Fig. 6, for both cases, instead of \#2, \#3 lateral flow strip with 
Fig. 5 Experimental results with metering and discharging: (a) metering or discharging sample volume respectively with water or urine sample; (b) two photographs of the immunoassay cassette before and after sample loading a

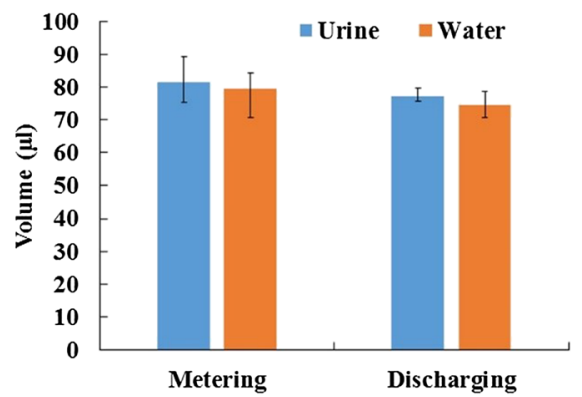

b

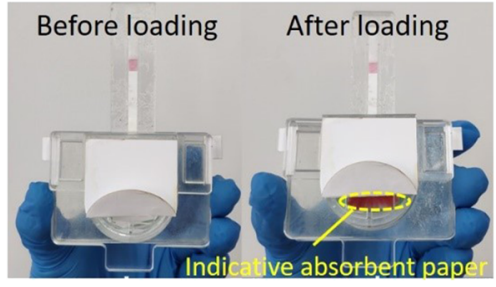

low-positive can be successfully detected. Compared to white LEDs, significantly higher signal reading with the test line can be achieved with green LEDs for different sample concentrations (\#3, \#5, and \#10 in Fig. 6), which demonstrate that higher detection sensitivity can be achieved with green LEDs. Therefore, a customized back light module with green light is adopted in the handheld reader.

Since the detection is performed in an enclosed box where the lateral flow strip is illuminated with a green lighting source, appropriate lighting conditions with a properly set driving current is critical to the detection performance. For comparison, experiments with different driving currents were performed. Standard gold colloid lateral flow strips, rather than the cassette, were used to evaluate the performance of the handheld reader itself. HIV serum samples with different concentrations were used in experiments. In each test, $80 \mu \mathrm{l}$ of HIV serum sample was applied onto the standard lateral flow strip and incubated for $15 \mathrm{~min}$. Figure 7 depicts the experimental results for three cases respectively with low $(0.5 \mathrm{~mA})$, medium (1.7 mA) and high (10.2 mA) driving currents. Error bars come from multiple repeated experiments.

As shown in Fig. 7, it can be found that with a medium driving current (1.7 $\mathrm{mA})$, optimal performance can be achieved compared to others. As shown in Fig. 7, when the driving current is too low or too high, \#3 lateral flow strip with

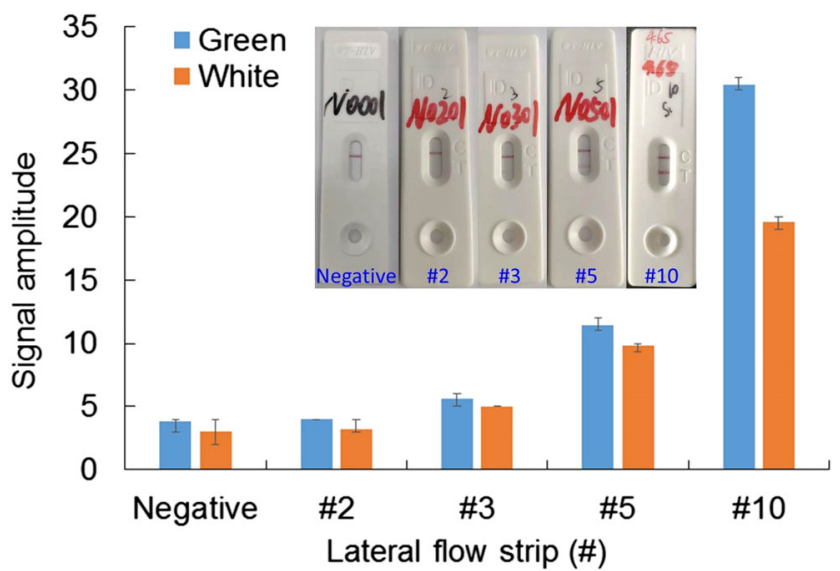

Fig. 6 Experimental results of lateral flow strip illumined with green or white light. Inset shows photographs of standard lateral flow strips taken by a smartphone camera low-positive cannot be successfully detected with acceptable credit. Inserts are the recovered photographs of the standard lateral flow strips with medium-positive (\#5) respectively with different driving currents, which are taken by the handheld optical reader with green light illumination. It has been demonstrated that optimal performance can be achieved with a medium driving current when the lateral flow strip is properly illuminated without saturation. It was found that comparable performance can be achieved when the driving current was between $1.5 \mathrm{~mA}$ and $1.8 \mathrm{~mA}$. Therefore, a medium driving current $(1.7 \mathrm{~mA})$ was applied in the handheld reader.

\subsection{Performance evaluation of immunoassay cassette for HIV test with urine sample}

The performance of the HIV immunoassay cassette was evaluated with HIV urine sample. HIV urine samples with different concentrations were used in experiments. In each test, more than $120 \mu \mathrm{l}$ urine sample was loaded into the described cassette ( $80 \mu \mathrm{l}$ was used in the test), and the lateral flow strip was detected with the described handheld optical reader after 15-min incubation, once the cassette was fully assembled. For comparison, parallel tests were performed with standard lateral flow strips. A benchtop immunoassay gold colloid reader (RTR-G100, Xiamen Innovax Biotech Co., Ltd) was used to detect the standard lateral flow strip after 15-min incubation once $80 \mu$ l of urine sample was applied. Figure 8 depicts the

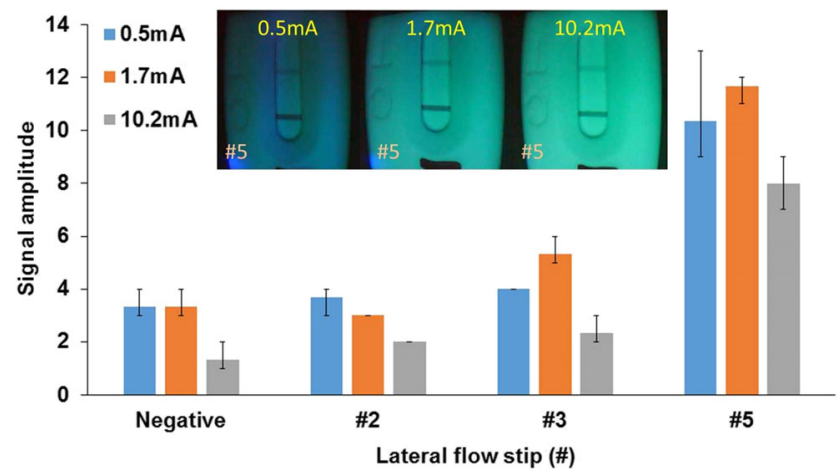

Fig. 7 Experimental results detected by the handheld optical reader with different driving currents. Inset shows recovered photographs of standard lateral flow strips taken by the handheld optical reader 


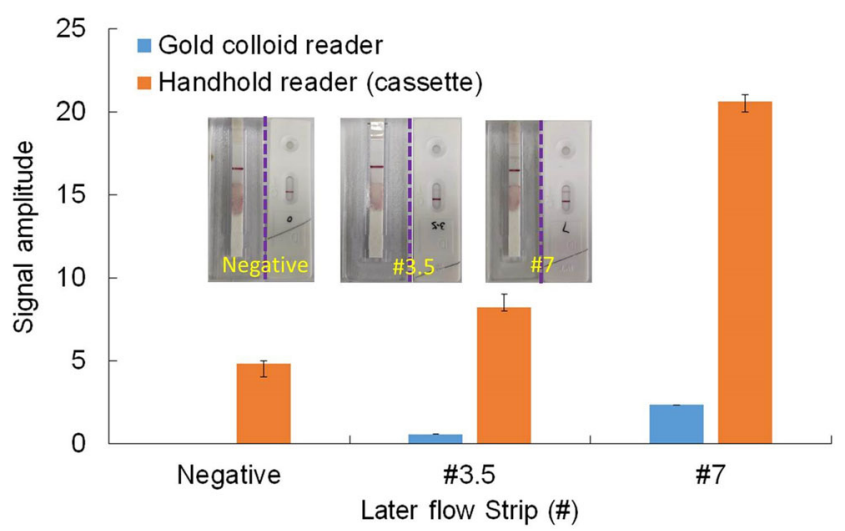

Fig. 8 HIV urine testing with both the described method and the standard lateral flow strip with a benchtop reader. Each inset shows photographs of lateral flow strips respectively from the described cassette (left) and the standard strip cassette (right)

experimental results for both cases respectively with the described cassette and the standard lateral flow strip. Error bars come from multiple repeated experiments. As shown in Fig. 8, for both methods, both low (\#3.5) and medium (\#5) positive samples can be successfully detected and differentiated from the negative one.

As shown in Fig. 8, acceptable detection sensitive with the described method can be achieved. Inserts are the photographs for both cases with detection to negative, the low (\#3.5) and medium (\#7) positive samples. For each insert (negative, \#3.5, and \#7), the left photograph is from the described cassette, while the right one is from the standard lateral flow strip. It has been demonstrated that comparable performance between the described method and the standard lateral flow strip with a benchtop reader can be achieved. However, both the cost and the size of the described handheld optical reader are significantly less than those of the benchtop reader, which is more suitable for POC diagnosis at resource-poor settings.

\section{Conclusions and outlook}

We describe a disposable, easy-to-operate microfluidic immunoassay cassette for HIV urine tests with a handheld optical reader for use at the point of care including home use. The HIV immunoassay cassette consists of three different modules respectively for sample introduction, metering and detection. The sample introduction module provides a user-friendly interface for users to conveniently add urine sample into the cassette. The metering module can adequately control the test sample volume with acceptable deviation. The detection module is able to perform HIV urine detection with an integrated lateral flow strip that uses gold colloid reporter. Once the cassette is fully assembled, the metering chamber will be fluidically connected from its bottom by a sharp needle from the detection module, which allows the urine sample to be continually aspirated by the sample absorbing pad of the lateral flow strip for immunoassay. The whole operation can be performed just by hand without any instrument or special equipment. After 15-min incubation, the cassette can be inserted into a handheld optical reader to detect the test result within $5 \mathrm{~s}$. A custom image processing algorithm was developed to search and locate the test and control lines of the lateral flow strip. The total cost of components of the handheld optical reader is around seventy dollars, which is suitable for POC diagnosis or home test.

Compared to the traditional test by just adding the urine sample to a lateral flow strip, there have a couple of advantages with the developed method. First, with the microfluidic cassette, the allowable test sample volume could be potentially increased for higher sensitivity. Second, there has no need for any sample metering tools since there has a metering chamber within the microfluidic cassette. Third, the risk of environmental contamination (including HIV sample contamination and even spread of the unfriendly smell from urine sample) can be reduced since both the lateral flow strip and the excess sample are sealed inside the cassette. Fourth, the detection sensitivity and accuracy can be improved by combining the cassette with the handheld optical reader compared to direct eye observation which could be unfavorably affected by the operator and the environment.

The performance for both the HIV immunoassay cassette and the handheld optical reader has been systematically evaluated. Consistent metering, discharging and applying ensure adequate and reproducible test sample volumes. To improve the system performance, green light instead of white light was adopted to illuminate the lateral flow strip. A proper working current for the green back light module was chosen to ensure the system performance. HIV antibody-spiked urine samples were successfully detected by the immunoassay cassette with a handheld optical reader. It has been demonstrated that comparable results can be achieved with the described method comparing to the standard lateral flow strip with a benchtop reader. However, simple, easy-to-operate, low-cost, and integrated HIV urine testing, which is highly desired for POC diagnosis or even home test, can be conveniently achieved with the described method based on a disposable cassette and a handheld, affordable optical reader.

Acknowledgements The work was supported by National Science and Technology Major Project (2018ZX10732101-001-009), the National Natural Science Foundation of China (No. 81871505, 81371711), the Fundamental Research Funds for the Central Universities (XK1802-4, PYBZ1830, PT1908), and the research fund to the top scientific and technological innovation team from Beijing University of Chemical Technology (No. buctylkjcx06).

\section{Compliance with ethical standards}

Conflict of interest The authors declare that they have no conflict of interest. 


\section{References}

Beijing Wantai Biological Pharmacy Enterprise Co. Ltd., User's manual: diagnosis kit for HIV-1 antibody test with urine sample (gold colloid lateral flow strip). VER 00 (2019)

Z. Chen, H. Zhu, D. Malamud, C. Barber, Y.Y. Serge Ongagna, R. Yasmin, S. Modak, M.N. Janal, W.R. Abrams, R.A. Montagna, A rapid, self-confirming assay for HIV: simultaneous detection of antiHIV antibodies and viral RNA. J. Aids Clin. Res. 7(1), 540-561 (2016)

X. Cheng, A. Gupta, C. Chen, R.G. Tompkins, W. Rodriguez, M. Toner, Enhancing the performance of a point-of-care CD4+ T-cell counting microchip through monocyte depletion for HIV/AIDS diagnostics. Lab Chip 9, 1357-1364 (2009)

K.P. Delaney, B.M. Branson, A. Uniyal, P.R. Kerndt, P.A. Keenan, K. Jafa, A.D. Gardner, D.J. Jamieson, M. Bulterys, Performance of an oral fluid rapid HIV-1/2 test: Experience from four CDC studies. Aids 20, 1655-1660 (2006)

K. Faulstich, R. Gruler, M. Eberhard, K. Haberstroh, Developing rapid mobile POC systems. Part 1: Devices and applications for lateralflow immunodiagnostics. IVD Technol. 13, 47-53 (2007)

G.B. Fogazzi, M.A. Perazella, The urinary sediment: An integrated view, $3^{\text {rd }}$ edition. Kidney Int. 78, 1202-1203 (2010)

A. Gaber, H. Hamed, E. Elsawy, Comparison between real-time polymerase chain reaction and DNA-microarray in detection and identification of mycobacterium species. Genet. Mol. Res. 16, 1-8 (2017)

T.C. Granade, S. Workman, S.K. Wells, A.N. Holder, S.M. Owen, C.-P. Pau, Rapid detection and differentiation of antibodies to HIV-1 and HIV-2 using multivalent antigens and magnetic Immunochromatography testing. Clin. Vaccine Immunol. 17(6), 1034-1039 (2010)

R. Hart, M. Mauk, C. Liu, X. Qiu, J. Thompson, D. Chen, D. Malamud, W. Abrams, H. Bau, Point-of-care oral-based diagnostics. Oral Dis. 17, 745-752 (2011)

U.M. Jalal, G.J. Jin, J.S. Shim, Paper-plastic hybrid microfluidic device for smartphone-based colorimetric analysis of urine. Anal. Chem. 89, 13160-13166 (2017)

M. Kingston, D. Bansal, E. Carlin, 'Shelf life'of Trichomonas vaginalis. Int. J. STD AIDS 14, 28-29 (2003)

E. Lepowsky, F. Ghaderinezhad, S. Knowlton, S. Tasoglu, Based assays for urine analysis. Biomicrofluidics 11, 051501 (2017)

C.-C. Lin, C.-C. Tseng, T.-K. Chuang, D.-S. Lee, G.-B. Lee, Urine analysis in microfluidic devices. Analyst 136, 2669-2688 (2011)
C. Liu, X. Qiu, S. Ongagna, D. Chen, Z. Chen, W.R. Abrams, D. Malamud, P.L. Corstjens, H.H. Bau, A timer-actuated immunoassay cassette for detecting molecular markers in oral fluids. Lab Chip 9, 768-776 (2009)

E. Mahoney, J. Kun, M. Smieja, Q. Fang, Point-of-care urinalysis with emerging sensing and imaging technologies. J. Electrochem. Soc. 167, 037518 (2020)

C.F. Paredes, I. Tellez, C. del Rio, Rapid HIV testing: A review of the literature and implications for the clinician. Curr HIV/AIDS Rep 3, 169-175 (2006)

S. Qian, H. Bau, Analysis of lateral flow bio-detectors: Competitive format. Anal. Chem. 326, 211-224 (2004)

X. Qiu, J.A. Thompson, Z. Chen, C. Liu, D. Chen, S. Ramprasad, M.G. Mauk, Y.Y. Serge Ongagna, C. Barber, W.R. Abrams, Finger-actuated, self-contained immunoassay cassettes. Biomed. Microdevices 11, 1175-1186 (2009)

X. Qiu, D. Chen, C. Liu, M.G. Mauk, T. Kientz, H.H. Bau, A portable, integrated analyzer for microfluidic - based molecular analysis. Biomed. Microdevices 13, 809-817 (2011)

V. Suresh, O. Qunya, B.L. Kanta, L.Y. Yuh, K.S. Chong, Non-invasive paper-based microfluidic device for ultra-low detection of urea through enzyme catalysis. R. Soc. Open Sci. 5, 171980 (2018)

C. Taramasco, T. Rodenas, F. Martinez, P. Fuentes, R. Munoz, R. Olivares, V.H.C. Albuquerque, J. Demongeot, A novel low-cost sensor prototype for nocturia monitoring in older people. IEEE Access. 6, 52500-52509 (2018)

X. Tian, J. Wang, D. Du, S. Li, C. Han, G. Zhu, Y. Tan, S. Ma, H. Chen, M. Lei, Medical imaging and diagnosis of subpatellar vertebrae based on improved Laplacian image enhancement algorithm. Comput. Methods Prog. Biomed. 187, 105082 (2020)

T. Vos et al., Global, regional, and national incidence, prevalence, and years lived with disability for 310 diseases and injuries, 1990-2015: A systematic analysis for the global burden of disease study 2015. Lancet 388, 1545-1602 (2016)

World Health Organization, HIV/AIDS, https://www.who.int/newsroom/fact-sheets/detail/hiv-aids (2019). Accessed 15 Dec 2019

J. Zhao, L. Chang, L. Wang, Nucleic acid testing and molecular characterization of HIV infections. Eur. J. Clin. Microbiol. Infect. Dis. 38, 829-842 (2019)

Publisher's note Springer Nature remains neutral with regard to jurisdictional claims in published maps and institutional affiliations. 